\title{
Multifractal Analysis of the Interaction between Chinese and American Stock Markets
}

\author{
Yanjun Qiu, Cheng Ye \\ Department of Statistics, College of Economics, Jinan University, Guangzhou, China \\ Email: qiustudent_r@163.com
}

How to cite this paper: Qiu, Y.J. and Ye, C. (2019) Multifractal Analysis of the Interaction between Chinese and American Stock Markets. Open Journal of Statistics, 9, 143-157. https://doi.org/10.4236/ojs.2019.91011

Received: January 28, 2019

Accepted: February 25, 2019

Published: February 28, 2019

Copyright $\odot 2019$ by author(s) and Scientific Research Publishing Inc. This work is licensed under the Creative Commons Attribution International License (CC BY 4.0).

http://creativecommons.org/licenses/by/4.0/

(c) (i) Open Access

\begin{abstract}
In this paper, we select yield series of the SSE index and the S \& P 500 index as the research object. Firstly, we take the financial crisis as the dividing point, and decompose the whole sample period into three periods: before the financial crisis, during the financial crisis, and after the financial crisis. Secondly, the degree of interaction between Chinese and American stock markets was tested and calculated in stages, and the cross-correlation relationship became more significant after the financial crisis. Then the MF-DCCA method is used to analyze the multifractal interaction of the whole period. It is found that the interaction relationship is multifractal in the short-term and long-term, and shows stronger in the short-term. In addition, the interaction relationship is persistent for small fluctuations in the short-term, and it is anti-sustainability in the case of large fluctuations; it is persistent in all fluctuations in the long run. Finally, the multifractal analysis was carried out for the three periods. It was found that during the financial crisis, the interaction had stronger multifractality and volatility, and the risk was higher.
\end{abstract}

\section{Keywords}

MF-DCCA, Financial Crisis, Interaction Relationship, Multifractal

\section{Introduction}

The United States has the largest GDP and is the largest developed country. China, with the world's second largest GDP, is the largest developing country. Studying the dynamic interaction between China and the US stock market, and discovering the degree of interaction between China and the United States, especially in the extreme case of financial crisis, it is beneficial for both governments to control financial risks under extreme circumstances. Especially in 2007, the financial crisis broke out and finally spread to the whole world. Therefore, stud- 
ying the multifractal relationship between the Chinese stock market and the US stock market will not only provide important suggestions for investors' investment, but also more favorable government under extremely adverse conditions control the financial risk, safeguard national security and stability.

Methods to analyze the correlation among different stock markets mainly include co-integration test, trend elimination volatility analysis (DFA), MF-DFA and DCCA combined multiple fractal trend elimination interactive correlation analysis (MF-DCCA), VAR, Granger causality test, impulse response, time-varying t-Copula model, and DCC-GARCH model; The research object includes the return rate and volatility of the stock index. From a regional perspective, the research object includes the stock market between different countries or regions and different stock exchanges in the same country; With the changes in methods and samples, the conclusions obtained are not completely consistent, but almost all the literatures have found that the "subprime mortgage crisis significantly enhances the linkage between different financial markets". Huang et al. (2000) used the co-integration test and found that there was no co-integration relationship between China's inland stock market and the stock markets of the United States, Japan, Taiwan, and Hong Kong [1]. Zhang and Li (2014) used the co-integration test method to find that even after the financial crisis broke out, there was no long-term co-integration relationship between the Chinese stock market and the US market [2]. Shi Daimin (2002) used the co-integration method to find a long-term stable linkage relationship in the Shanghai and Shenzhen stock markets [3]. Chen Shoudong et al. (2003) used the Granger causality test and the GARCH-M model to find that the yields of the Shanghai and Shenzhen stock markets are Granger factors to each other, and there is a strong correlation [4]; however, there is only a volatility spillover effect from Shenzhen to Shanghai. Gao Ying, Li Lili (2008) used the VAR, Granger causality test, impulse response and Johansen co-integration test method to test and analyze the CSI 300 Index and the world's major stock indices from 2005 to 2006, and found that in addition to the Nikkei index, the CSI 300 Index has a long-term co-integration relationship with other major indices [5]. Xu Youjun et al. (2010) used the DCC-GARCH model to study the linkage between China and international stock markets from 1997 to 2009. It was found that before the financial crisis, the linkage between China and international stock markets was weak, and the financial crisis strengthened this linkage [6]. Gong Pu and Huang Rongbing (2009) used the time-varying $\mathrm{t}$-Copula model to measure the impact of the subprime mortgage crisis on the mainland stock market. They found that the outbreak of the subprime mortgage crisis was transmitted to the mainland stock market through the Hong Kong stock market, which aggravated the shock of the mainland stock market [7]. Fang Yi, Gui Peng (2010) used the Granger causality test method and the variance decomposition method to find that after the subprime mortgage crisis in the Asia-Pacific region, the independence was significantly reduced, while the linkage was further strengthened [8]. Zhang Bing and Fan Zhizhen (2010) analyzed the long-term equilibrium relationship between the 
Chinese and American stock markets using the Johansen cointegration test and the DCC-GARCH model. They found that there is no long-term equilibrium relationship between these two markets, and Chinese stock market only has a weak guiding effect on American stock market; however, after the implementation of QDII, the volatility spillover effect between us stock market and the Chinese stock market keeps increasing [9]. Podobnik and Stanley (2008) introduced the trend elimination volatility analysis method (DFA) into the interactive cross-correlation analysis and proposed the trend elimination cross-correlation analysis method (DCCA) [10]. Zhou (2008) combined MF-DFA and DCCA methods to test the cross-correlation relationship between two non-stationary time series and proposed the multifractal elimination trend cross-correlation analysis (MF-DCCA) method [11]. Since the introduction of multifractal elimination trend cross-correlation analysis (MF-DCCA), it has attracted the interest of many scholars. For example, Ma and Wei (2013) used the MFDXA method to study the interaction between China's stock market and neighboring stock markets such as Japan, South Korea, and Hong Kong, and found that the interaction between China's stock market and the stock markets of these neighboring countries has strong multifractal characteristics; in addition, the cross-correlation relationship shows persistence for small fluctuations in the short term, while large fluctuations show anti-sustainability [12].

In summary, in China, the current literature is mainly based on the traditional linear method to study the linkage relationship of the stock market, and less use of multifractal theory to study the linkage relationship between stock markets. However, in foreign countries, such as Wang (2010) [13], Peters (1994) [14] found that the financial market is a nonlinear dynamic complex system with multiple fractals and chaotic structures. Traditional linear models, such as VAR and co-integration models, cannot be used to describe the nonlinear dynamic interactive correlation between stock markets. Moreover, the interaction between stock market volatility sequences will also change with time. Therefore, the linear bivariate GARCH family model, such as DCC-GARCH and BEKK-GARCH models, cannot well capture the dynamic interactive relationship between stock market volatility. Indicates, when using the traditional linear model to analyze the financial market, there may be a large deviation.

In view of the above shortcomings, this paper uses the nonlinear dynamic multifractal elimination trend cross-correlation analysis (MF-DCCA) method to study the multifractal characteristics of the cross-correlation between China's Shanghai Stock Index and the US Standard \& Poor's Index. Taking the outbreak period of the financial crisis as the dividing point, the whole sample period is divided into three periods: before the crisis, during the crisis period and after the crisis. The dynamic interaction correlation coefficient between the two markets in the three periods is calculated and the significance test is carried out. The multifractal characteristics of the interactions between the three periods are analyzed from the perspective of the cross-correlation multifractal spectrum width and the multifractal spectrum. 


\section{Methodology}

\subsection{Cross-Correlation Test}

Podobnik et al. (2009) [15] proposed a new cross-correlation test to test whether the cross-correlation of two sequences is significant. Suppose $\left\{x_{i}, i=1,2, \cdots, N\right\}$ and $\left\{y_{i}, i=1,2, \cdots, N\right\}$ are two time series of the same length $N$. Formulas are defined as follows:

$$
\begin{gathered}
C_{i}=\frac{\sum_{k=i+1}^{N} x_{k} y_{k-i}}{\sqrt{\sum_{i}^{N} x_{k}^{2} \sum_{i}^{N} y_{k}^{2}}} \\
Q_{c c}(m)=N^{2} \sum_{i=1}^{m} \frac{C_{i}^{2}}{N-i} .
\end{gathered}
$$

The cross-correlation test statistic $Q_{c c}(m)$ approximately obeys $\chi^{2}(m)$ distribution, The interaction correlation between the two sequences is significant if the interaction correlation test statistic exceeds the critical value of the chi-square distribution.

According to Equation (1), the calculation principle of lag correlation coefficient $C_{i}$ and horizontal correlation coefficient is the same, it can only measure the linear correlation of stationary sequences. In order to overcome the above shortcomings, Zebende (2011) proposed a DCCA interaction correlation coefficient method [16]. This method can effectively measure the correlation between stationary and non-stationary sequences. The cross correlation coefficient is defined as the ratio of the detrend covariance function $F_{D C C A}^{2}$ to the detrend variance function, i.e., Equation (3).

$$
\rho_{D C C A}(s)=\frac{F_{D C C A}^{2}(s)}{F_{D F A\{x(i)\}(s)} F_{D F A\{y(i)\}(s)}} .
$$

\subsection{Multiple Cross-Correlation Analysis}

Given two time series $\{x(i)\}$ and $\{y(i)\}, i=1,2, \cdots, N, N$ are the lengths of this time series, the MF-DCCA method can be described as follows:

Step 1: Generate a cumulative dispersion sequence $\{X(i)\}$ and $\{Y(i)\}$ :

$$
X(i)=\sum_{t=1}^{i}(x(t)-\bar{x}), \quad Y(i)=\sum_{t=1}^{i}(y(t)-\bar{y}), i=1,2, \cdots, N .
$$

where $\bar{x}=\frac{1}{N} \sum_{i=1}^{N} x(i), \bar{y}=\frac{1}{N} \sum_{i=1}^{N} y(i)$.

Step 2: Divide new sequences into $N_{s}$ intervals, each of which is continuous and non-overlapping with length $s$. Since $N$ is not necessarily an integer multiple of $s$, in order to make full use of the remaining data of the tail, the same operation is repeatedly performed from the end, thereby obtaining the $2 N_{s}$ intervals. We set the window $s$ range to $10<s<N / 4$. This paper uses a total of 15 years of data from 2002 to 2017, where $N$ is the sample size. Considering the short-term behavior and the need for a suitable number of samples per interval to calculate 
the covariance function, we set the lower limit of $s$ to 10; In addition, considering that N/4 means about 45 months, the long-term relationship between the two sequences can be sufficiently included, so we set the upper limit of $s$ to N/4. In fact, there is no significant difference in results, regardless of whether the upper limit is larger or the lower limit is smaller.

Step 3: Fit the local trend function in each subinterval $v, v=1,2, \cdots, 2 N_{s}$ by least squares method and get the fitted polynomial $\tilde{X}_{v}(i), \tilde{Y}_{v}(i)$. Then, the detrend covariance function can be calculated. For each subinterval $v, v=1, \cdots, N_{s}$, the covariance function is calculated as follows:

$$
F^{2}(s, v)=\frac{1}{s} \sum_{i=1}^{s}\left|X((v-1) s+i)-\tilde{X}_{v}(i)\right| \cdot\left|Y((v-1) s+i)-\tilde{Y}_{v}(i)\right| .
$$

and for each subinterval $v, \quad v=N_{s}+1, \cdots, 2 N_{s}$, the covariance function is calculated as follows:

$$
F^{2}(s, v)=\frac{1}{s} \sum_{i=1}^{s}\left|X\left(N-\left(v-N_{s}\right) s+i\right)-\tilde{X}_{v}(i)\right| \cdot\left|Y\left(N-\left(v-N_{s}\right) s+i\right)-\tilde{Y}_{v}(i)\right| \cdot(6)
$$

Step 4: Calculate the $q$-order detrend covariance function. For $q \neq 0$, the $q$-order detrend covariance function is defined as follows:

$$
F_{x y}(q, s)=\left\{\frac{1}{2 N_{s}} \sum_{v=1}^{2 N_{s}}\left[F^{2}(s, v)\right]^{q / 2}\right\}^{1 / q},
$$

and for $q=0$,

$$
F_{x y}(0, s)=\exp \left\{\frac{1}{4 N_{s}} \sum_{v=1}^{2 N_{s}} \ln \left[F^{2}(s, v)\right]\right\} .
$$

Finally, we show a double logarithmic plot of $F_{x y}(q)$ versus $s$ at each fixed $q$. If two time series $\{X(i)\}$ and $\{Y(i)\}$ are long-range cross-correlation, $F_{x y}(q, s)$ will increase with $s$ in power law,

$$
F_{x y}(q, s) \sim s^{h_{x y}}(q)
$$

The above formula can also be expressed as

$$
\log F_{x y}(q, s)=h_{x y}(q) \log (s)+\log A
$$

where $h_{x y}(q)$ is the scale index, describes the power-law relationship of two time series. In particular, when $q=2$, especially when time series $\{X(i)\}$ is the same as $\{Y(i)\}$, MF-DCCA method is MFDFA, otherwise, MF-DCCA is the standard DCCA method. When $h_{x y}(q)>0.5$, the interaction exhibits long-range persistence; and when $h_{x y}(q)<0.5$, the cross-correlation exhibits anti-persistence; if $h_{x y}(q)=0.5$, there is no interaction or at most short-range interaction between the sequences.

Yuan and Zhuang (2009) proposed that $\Delta h=h_{\max }(q)-h_{\min }(q)$ can be used to measure multifractal strength. The larger $\Delta h$, the stronger the multi-fractality and the greater the market risk [17]. So according to this idea, use $h_{x y}(q)$ instead of $h(q)$, then $\Delta h_{x y}$ can be used to measure the multifractal strength of the cross-correlation between the two sequences and the risk of the market. 
Shdkhoo and Jafari (2009) propose an interaction correlation index [18]. The relationship between $h_{x y}(q)$ and multifractal quality index $\tau_{x y}(q)$ is as follows:

$$
\tau_{x y}(q)=q h_{x y}(q)-1 .
$$

If $\tau_{x y}(q)$ is a linear function of $q$, then the interaction between the two sequences is single-fractal, otherwise it is multifractal. Carry out the Legendre transformation on the Equation (11) to obtain the following relationship:

$$
\begin{gathered}
\alpha_{x y}=\tau_{x y}^{\prime}(q)=h_{x y}(q)+q h_{x y}^{\prime}(q) . \\
f_{x y}=q \alpha_{x y}-\tau\left(\alpha_{x y}\right)=q\left(\alpha_{x y}-h_{x y}(q)\right)+1 .
\end{gathered}
$$

Among them, $f_{x y}(q)$ is a multifractal spectrum, and $\alpha$ is a singular index, which is used to describe the singularity of each interval. The smaller the $\alpha$, the larger the singularity, vice versa.

\section{Empirical Results}

\subsection{Data}

In this paper, we select the US Standard \& Poor's S \& P 500 Index and the daily closing price data of the Shanghai Composite Index as the research object, from January 4, 2002 to March 14, 2017. After excluding different trading days, we have 3561 samples. Since the global financial crisis broke out between 2007 and 2008, January 1, 2007 and December 31, 2008 were set as the time points for segmentation. The full sample period is divided from January 4, 2002 to December 29, 2006, January 1, 2007 to December 31, 2008, and January 5, 2009 to March 14, 2017 which representing the period before, during and after the crisis. The logarithmic yields of these two indices are shown in Figure 1. We use a logarithm with a base of 2 here.

It can be seen from Figure 1 that both yield series exhibit the characteristics of volatility aggregation, that is, large fluctuations are followed by large fluctuations, and small fluctuations are followed by small fluctuations. It can also be found that the SSE index yield series is more volatile than the S \& P500, which means that the SSE index yield sequence is more risky. Table 1 gives descriptive statistics of the yield series of two indices in three subsamples. In general, sample
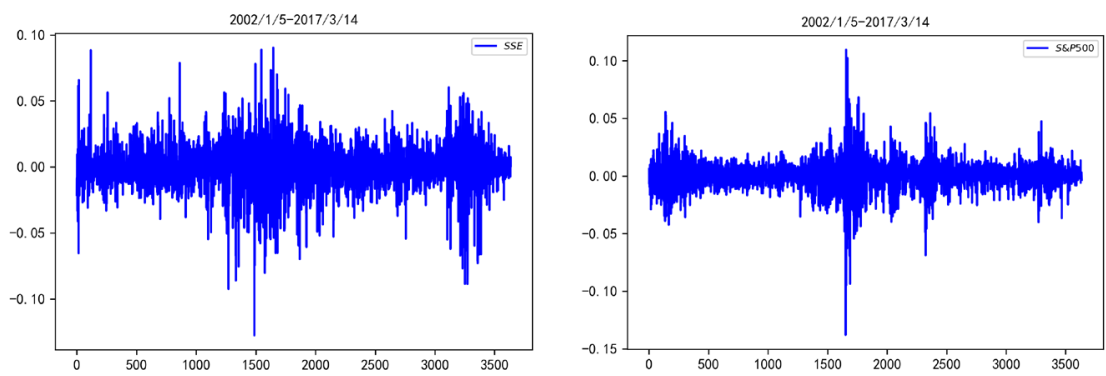

Figure 1. Daily yield of SSE index and S \& P 500 index. In this paper, we use SSE to represent the Shanghai Composite Index and S \& P500 to represent the Standard \& Poor's Index. 
Table 1. Descriptive statistics of SSE index and S \& P 500 yield series.

\begin{tabular}{|c|c|c|c|c|c|c|c|}
\hline & Mean & $\operatorname{Max}$ & Min & S.D & Ske & Kur & $J-B$ \\
\hline \multicolumn{8}{|c|}{ Sample 1: January 5, 2002-December 29, 2006} \\
\hline SSE & 0.0004 & 0.0885 & -0.0654 & 0.0139 & 0.6523 & 6.97 & $845.38^{* * *}$ \\
\hline S \& P 500 & 0.0002 & 0.0557 & -0.0424 & 0.0103 & 0.1588 & 6.19 & $497.76^{\star * *}$ \\
\hline \multicolumn{8}{|c|}{ Sample 2: January 4, 2007 to December 31, 2008} \\
\hline SSE & -0.0008 & 0.0903 & -0.1276 & 0.0267 & -0.3973 & 4.76 & $73.44^{* * *}$ \\
\hline S \& P 500 & -0.0010 & 0.1096 & -0.1380 & 0.0205 & -0.6051 & 12.44 & $1782.57^{* * *}$ \\
\hline \multicolumn{8}{|c|}{ Sample 3: January 5, 2009-March 14, 2017} \\
\hline SSE & 0.0003 & 0.0604 & -0.0887 & 0.0155 & -0.7712 & 7.48 & $1794.32^{* * *}$ \\
\hline S \& P 500 & 0.0005 & 0.0684 & -0.0670 & 0.0111 & -0.1460 & 8.08 & $2073.54^{* * *}$ \\
\hline
\end{tabular}

Note: ${ }^{* *}$ indicates rejection of the null hypothesis at the $1 \%$ significance level; The symbols "Mean", "Max", "S.D”, "Ske”, "Kur" indicate mean, maximum, minimum, standard deviation, skewness, kurtosis, respectively; JB represents the Jarque-Bera statistic.

2, i.e., the period of financial crisis, the standard deviation of the two index returns is greater than the other two periods; The skewness of the three sub-sample is not zero, and the kurtosis is greater than 4 , indicating that during these three periods, the two yield series have the characteristics of sharp peaks and thick tails; The J-B statistic for both yield series is significant at the $1 \%$ level and therefore does not obey the normal distribution for the three periods. We mainly use Ske, Kur, S.D, and J-B indicators to describe the sequence, these formulas are as follows:

$$
\begin{gathered}
\text { Kur }=\frac{\frac{1}{n} \sum_{i=1}^{n}\left(x_{i}-\bar{x}\right)^{4}}{\left(\frac{1}{n} \sum_{i=1}^{n}\left(x_{i}-\bar{x}\right)^{2}\right)^{2}}, \text { Ske }=\frac{\frac{1}{n} \sum_{i=1}^{n}\left(x_{i}-\bar{x}\right)^{3}}{\left(\frac{1}{n} \sum_{i=1}^{n}\left(x_{i}-\bar{x}\right)^{2}\right)^{3 / 2}}, \\
S . D=\sqrt{\frac{1}{n} \sum_{i=1}^{n}\left(x_{i}-\bar{x}\right)^{2}}, J-B=\frac{n}{6}\left[S k e^{2}+\frac{(K-3)^{2}}{4}\right]
\end{gathered}
$$

where $x_{i}$ represents the $i$-th sample value of a specific sequence.

\subsection{Cross-Correlation Coefficient and Test}

Figure 2 shows the logarithmic plot of the cross-correlation test statistic for the different periods of the SSE yield series and the S \& P 500 index yield series. The ordinate is the logarithm of the cross-correlation test statistic, i.e., the logarithm of Equation (3). The abscissa is the logarithm of the degree of freedom $\mathrm{m}$ (from 1 to 1000). The critical value of the chi-square test at the $5 \%$ significance level is also shown in Figure 2. In order to better display the graph, we use the logarithm with a base of 10 .

It can be seen from Figure 2 that the $Q_{c c}(m)$ curve of the cross-correlation test statistic for the full sample period (2002/1/4-2017/3/14) is almost above the critical value, thus, the null hypothesis that suppose no cross-correlation is rejecting. 

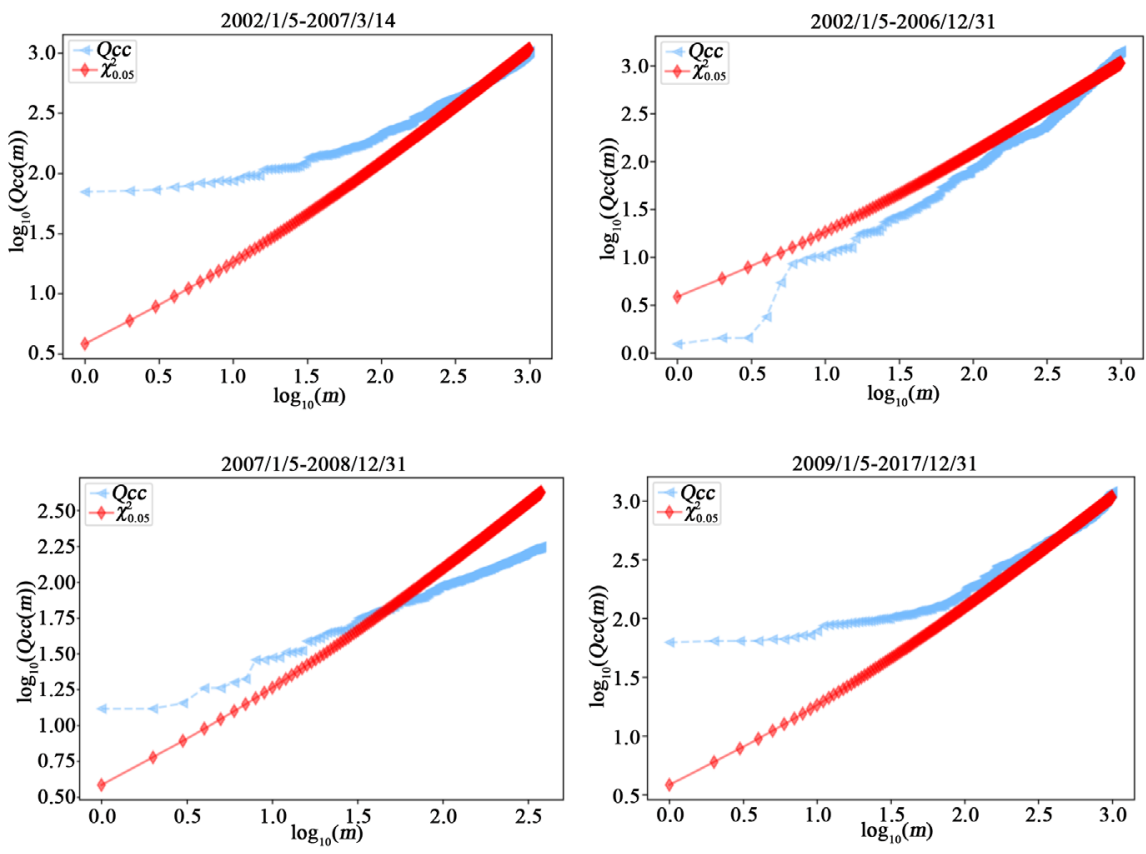

Figure 2. Interaction correlation test statistic $Q_{c c}(m)$.

That is, the SSE Index and the S \& P 500 Index have significant long-range interactions throughout the sample period. However, before the 2007 financial crisis, sample 1, the cross-correlation test statistic for the two yield series was below the critical value curve, so the cross-correlation of the two indices was weak before the financial crisis. During the financial crisis, sample 2, whether the cross-correlation of these two sequences is significantly related to the degree of freedom. When $\log _{10}(m)<1.8$ or $m<63$, the cross-correlation test statistic $Q_{c c}(m)$ curve is above the critical value curve; however, if $m>63$, the opposite is true. It shows that if the lag period is too long, there is no significant linkage between the Chinese and US stock markets, but it is highly correlated in the short term. After the financial crisis, sample 3, the $Q_{c c}(m)$ curves of these two sequences are almost all above the critical value curve. In summary, the correlation between the two sequences is gradually enhanced, from the weak correlation before the crisis to the short-term correlation during the crisis. In particular, the outbreak of the financial crisis has significantly strengthened the linkage between the two stock markets in China and the United States, which is consistent with the conclusions of most documents.

The test above can only analyze whether there is linear interaction correlation and if it's significant. In order to investigate the nonlinear cross-correlation of the two index's yield series in three subsamples, we use Equation (3) to calculate the correlation coefficient of detrending and volatility. The correlation coefficients before, during and after the financial crisis were 0.4761, 0.5337 and 0.5529 , respectively. This result further illustrates that the correlation of the two sequences in the three subsamples is gradually enhanced. The reason may be the outbreak of the financial crisis, which has led to panic psychological expectations 
of investors and the herd effect of investors. This negative impact has spread to other countries' markets, leading to a significant increase in the relevance of financial markets in countries during the crisis.

\subsection{Multifractal Analysis of China and American Stock Market}

The above results show that there is a significant interaction between the Shanghai Securities Composite Index yield series and the S \& P 500 index yield series during the whole sample period, crisis period and crisis; however, it cannot further explain how the long-term interaction of the two sequences is and whether this interaction is single-fractal or multifractal. Are the multifractal characteristics of interactions the same in the short and long term? To this end, the MF-DCCA method is used to examine the specific characteristics of the interaction relationship.

Figure 3 is a double logarithmic plot of the covariance function and the time scale plotted by the MF-DCCA method, $q=-10,-8, \ldots, 10$, the order of the fitted polynomial is 2, $x$ represents the Shanghai Securities Composite Index, and $y$ represents Standard \& Poor's Index. It can be seen from Figure 3 that when $\mathrm{q}$ takes different values, the $\log$-covariance function $\log _{10} F_{x y}(q)$ will change with the change of $\mathrm{q}$, so the interaction relationship between the two indices has multi-fractal features and multiple fractal dimensions. Secondly, although there is no simple linear relationship between $\log _{10} F_{x y}(q)$ and $\log _{10}(s), \log _{10} F_{x y}(q)$ linearly increases with the increase of $\log _{10}(s)$ from the trend or the whole. Therefore, there is a long-range power law interaction between the Shanghai Securities Composite Index and the Standard \& Poor's Index. Note that in order to better display the graph, we use a logarithm with a base of 10 here.

Ma and Wei (2013) pointed out that if there is a time scale $s^{*}$, when $s>s^{*}$, the linear trend or slope of the double logarithm graph changes or even structurally changes, and then this point is called crossover point. This crossover point $s^{*}$ can be used to distinguish the long-term and short-term nature of the time

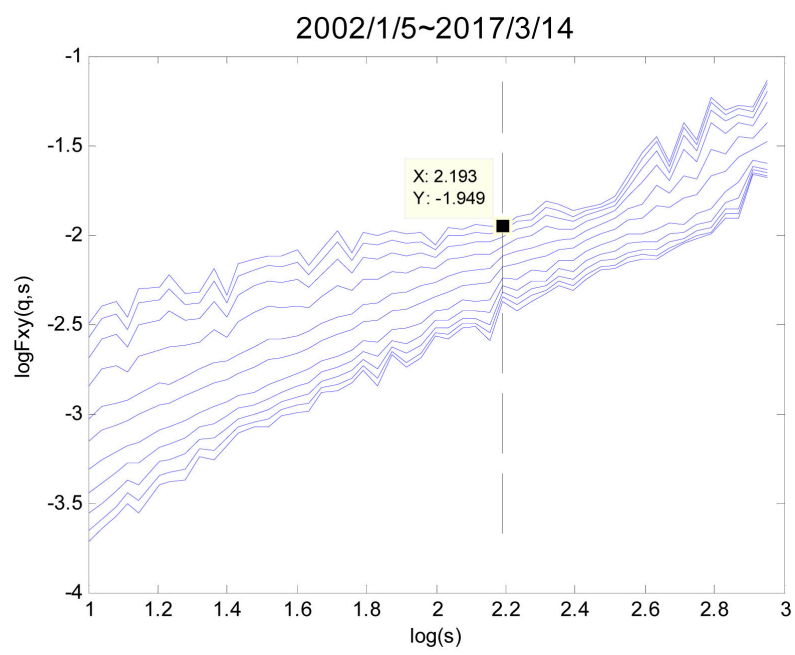

Figure 3. Double logarithmic plot of covariance function. 
series. The scale index for $s<s^{*}$ indicates short-term interaction behavior, which is easily affected by external factors of the market, such as political factors and economic factors; the scale index of $s^{*}$ indicates long-term interaction behavior, which is mainly determined by internal factors of time series [12]. Figure 3 shows that there is such a crossover point $s^{*}$ makes the slope of the linear relationship change on this time scale, which is roughly located at $\log _{10}\left(s^{\star}\right)=2.193$, or $s^{*}=156$. Specifically, when the time scale $s$ is small, the covariance function of different $\mathrm{q}$ is very divergent; as s becomes larger, the covariance functions of different q become closer and closer until $\log _{10}\left(s^{*}\right)=2.193$, That is, when $s^{*}=156$, the covariance functions of different $q$ have the smallest difference; however, when $s>156$, the covariance function of different $q$ becomes divergent again.

Figure 4 further shows the slopes of $\log F_{x y}(q)$ and $\log (s)$ at two scales, i.e. the cross-correlation index $h_{x y}(q)$ at different $q$, including the generalized Hurst exponent of the Shanghai Securities Composite Index yield series, and that of the Standard \& Poor's yield series, as well as the average Hurst exponent of the two sequences.

Figure 4 contains a very rich amount of information. First, whether in the long run or in the short term, the cross-correlation index $h_{x y}(q)$ depends on the $q$ value and changes with the $q$ value, which further illustrates the interaction between the Shanghai Securities Composite Index and the S \& P index yield series. The relationship is multifractal. Second, in the case of small scales $\left(s<s^{*}\right)$ or short-term, the cross-correlation index varies more, from about 0.7 to $0.3, h_{x y}$ $=0.4143$; in the case of large scales $\left(s>s^{*}\right)$, the cross-correlation index $h_{x y}(q)$ is less dependent on $\mathrm{q}$, and the range of variation is smaller and more stable in a long period of time and $h_{x y}$ is only 0.1338 . These two results show that the multifractal intensity of interaction in the short-term is more complicated than that of the long-term and multifractal features; while the interaction in the long-term is simpler, but it has stronger persistence (when $\mathrm{q}$ changes, $\mathrm{H}_{\mathrm{xy}}$ is close to 0.6 or greater than 0.6, and no less than 0.5). Obviously, this is in line with reality. In the long run, the China-US stock market is mainly affected by international common factors and its long-term running trends and laws. These factors have far-reaching and stable effects on the two stock markets, so long-term interaction relationships are also more persistent and stable. And being more stable
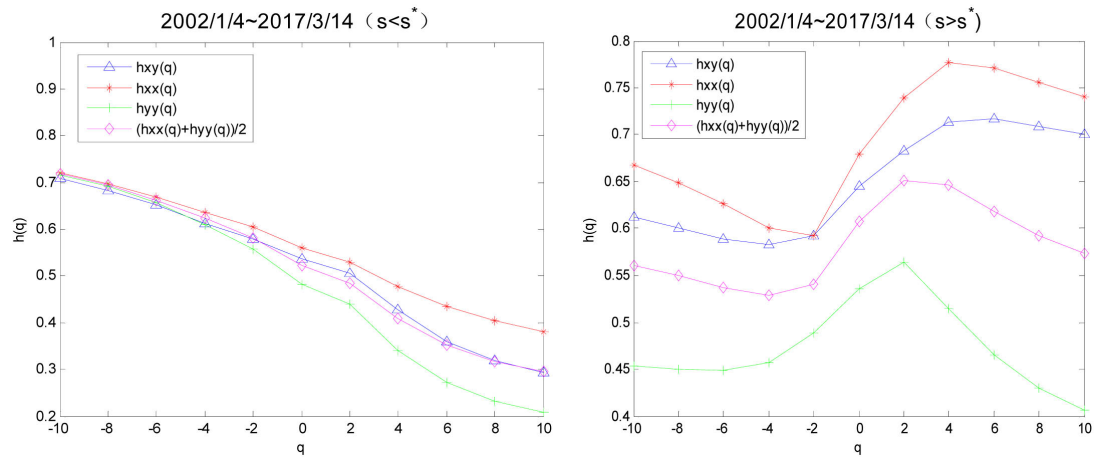

Figure 4. $h(q) \sim q$ of the Shanghai securities composite index and standard \& poor's index. 
means that the interaction is simpler and the strength of multiple fractals is relatively shorter in the short term. In the short-term, besides being affected by common factors, they are more affected by their domestic macroeconomic, policies, and behavioral habits of investors. Although there are certain interrelationships in the short term, they are affected by their specific factors. This interaction is not stable and the persistence is weaker but at the same time, it is more complex and multifractal features are more obvious.

Figure 4 (right) inadvertently answers the debate over whether the US stock market has long-term memory. It can be seen from the figure that the Hurst index of the Standard \& Poor's index, i.e. $h_{x x}(q)$, not only fluctuates less, but also fluctuates around 0.5; on the contrary, the Hurst index of the Shanghai Securities Composite Index, $h_{x x}(q)$, not only fluctuates greatly, but is much larger than 0.5 with the minimum value of around 0.6. This result shows that the Standard \& Poor's index has no long-term memory. The US stock market represented by the Standard \& Poor's index is more efficient and more in line with the efficient market hypothesis. The Shanghai Securities Composite Index has long-term memory, and its market pricing efficiency is lower, and is closer to fractal market hypothesis.

\section{Multifractal Analysis in Different Periods}

In the third part, we explore the multifractal features of the interaction between the Shanghai Composite Index and the Standard \& Poor's Index in the whole sample period. But whether the multifractal features remain the same in different periods is worth discussing. In order to fully describe the similarities and differences of the multifractal features in the three sample periods, we further adopt the width of the multifractal spectrum $\Delta \alpha$, the difference of the fractal dimension $\Delta f$ and the fractal quality index $\tau(q)$.

The singularity index $\alpha$ indicates the degree of local singularity of a subset of the system. The smaller $\alpha$ depicts the singularity of the large undulating point set, while the larger $\alpha$ depicts the singularity of the small undulating point set of the system. The width of the singular exponent, i.e. the width of the multifractal spectrum $\Delta \alpha$, represents the difference between the singularities of the system, and also describes the degree of multifractality of the system. The lager the $\Delta \alpha$ is, the more significant the multifractality of the system is. $f(\alpha)$ is used to measure the fractal dimension of the point set with singular exponent. The average fractal dimension of the large and small undulating point sets is $f_{l}$ and $f_{s}$ respectively, and the difference of them is $\Delta f . \Delta f$ can approximately depicts the frequency ratio of large fluctuations and small fluctuations of the system. The larger the $\Delta f$ is, the more frequent the large fluctuations of the system is.

Figure 5 and Figure 6 show the multifractal quality index and multifractal spectrum for three periods, respectively. It can be seen from the different periods in Figure 5 that before the financial crisis, the fractal quality index is almost linear with q; while during the financial crisis, the curve of $\tau(q)$ is more convex 


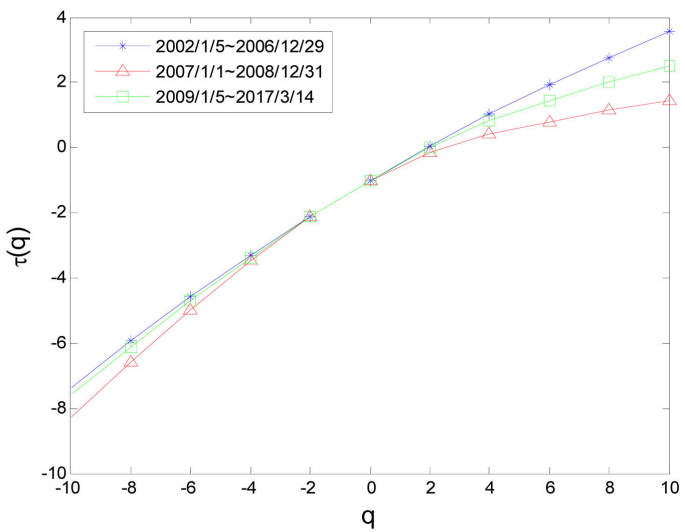

Figure 5. Values of $\tau(q)$ at different $q$.

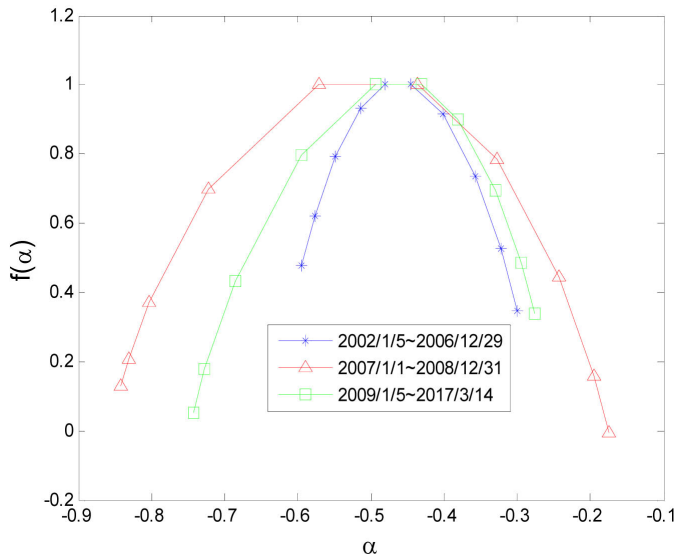

Figure 6. Values of $f(\alpha)$ at different $\alpha$.

and curved than the other two periods. After the crisis, the fractal quality index is in the middle of the other two periods. This shows that the interactions during this period have stronger multifractal characteristics. In addition, in Table 2, during the financial crisis, the multifractal spectral width between the two sequences $\Delta \alpha$ is 0.6660 , which is wider than the other two periods. Because the width of the multifractal spectrum is the indicator of the multifractal intensity and volatility of the cross-correlation relationship, which means the wider the multifractal spectrum, the greater the multifractal intensity and volatility. Therefore, during the financial crisis, the interaction between the two rates of return has a stronger multi-fractal and volatility. Figure 6 shows almost the same conclusion as Figure 5. During the crisis, the multifractal spectrum has a wider width, while before the crisis the width is smallest, and the post-crisis width is somewhere in between. It can also be seen from Table 2 that during the financial crisis, the extreme difference of the fractal dimension $\Delta f$ reaches 1.061, which is larger than the other two periods. Since the multifractal spectrum $f(\alpha)$ can characterize the complexity of each part with the same singularity index, usually the larger the absolute value of $\Delta f=f_{\max }-f_{\text {min }}$, the higher the degree of local fluctuation of the time series, and the more uneven the fluctuation 
Table 2. Multifractal features of three subsample intervals.

\begin{tabular}{cccc}
\hline & $2002 / 1 / 5-2006 / 12 / 29$ & $2007 / 1 / 1-2008 / 12 / 31$ & $2009 / 1 / 5-2017 / 3 / 14$ \\
\hline$\Delta \alpha=\alpha_{\max }-\alpha_{\min }$ & 0.2954 & 0.6660 & 0.4666 \\
$\Delta f=f_{\max }-f_{\min }$ & 0.6547 & 1.0061 & 0.9473 \\
\hline
\end{tabular}

distribution. Furthermore, during the crisis, violent fluctuations are relatively small but more frequent; and before the crisis, small fluctuations occur more frequently than violent fluctuations. This result shows that during the financial crisis, the interaction between the Chinese and American stock markets is more volatile, and a higher level of risk occurs in this period.

\section{Conclusions}

This paper applies MF-DCCA method to the Chinese SSE Index Yield Series and the US Standard \& Poor's S \& P 500 Index Yield Series, and the following conclusions were obtained:

First, compared with pre-crisis and post-crisis, the average cross-correlation coefficient between Chinese and American stock markets is the largest and very significant during the financial crisis; after the financial crisis, the average cross-correlation coefficient of the two sequences is higher than that of financial while it was bigger before the crisis. This shows that after the financial crisis, the interaction between the stock markets of China and the United States has become stronger.

Second, both in the short-term and in the long-term, the cross-correlation and autocorrelation of the two yield series exhibit multifractal characteristics. Both the cross-correlation and autocorrelation in the short-term show stronger multifractal. However, the long-term interaction correlation is stronger than the short-term.

Third, during the financial crisis, the interaction between China and the US stock market showed stronger multifractal characteristics. During the financial crisis, the multifractal spectrum width and range were larger than that in the other two periods, indicating that the volatility of the interaction between China and the US stock market is significantly increased, and the degree of volatility is chaotic, and the degree of risk is also increasing in the period of financial crisis.

In short, in a long period before the subprime mortgage crisis, China's stock market and the international financial market are not closely linked; however, since the subprime mortgage crisis, the linkage between China's stock market and international financial market has significantly increased. Therefore, with the improvement of the relevant market system in China's stock market and the acceleration of the pace of internationalization, the shocks may be fiercer in the future. How to deal with these shocks and to maintain the stability of China's stock market will become an increasingly important research topic. 


\section{Conflicts of Interest}

The authors declare no conflicts of interest regarding the publication of this paper.

\section{References}

[1] Huang, B.-N., Yang, C.-W. and Hu, J.W.-S. (2000) Causality and Cointegration of Stock Markets among the United States, Japan, and the South China Growth Triangle. International Review of Financial Analysis, 9, 281-297. https://doi.org/10.1016/S1057-5219(00)00031-4

[2] Zhang, B. and Li, X.-M. (2014) Has There Been Any Change in the Comovement between the Chinese and US Stock Markets? International Review of Economics \& Finance, 29, 525-536. https://doi.org/10.1016/j.iref.2013.08.001

[3] Shi, D.M. (2002) Cointegration Research on the Volatility of Stock Index in Shanghai and Shenzhen Stock Markets. The Journal of Quantitative \& Technical Economics, No. 9, 103-105.

[4] Chen, S.D., Chen, L. and Liu, Y.W. (2003) Correlation Analysis of China's Shanghai and Shenzhen Stock Market Returns and Its Volatility. Journal of Financial Research, No. 7, 80-85.

[5] Gao, Y. and Jin, L.L. (2008) Analysis of the Correlation between the Shanghai and Shenzhen 300 Index and the World's Major Stock Indices. Business Review, 20, 3-8.

[6] Xu, Y.J., Wang, X.X. and Jia, J.J. (2010) Analysis of the Linkage between Chinese Stock Market and International Stock Market. Economic Survey, No. 5, 124-128.

[7] Gong, P. and Huang, R.B. (2009) An Empirical Analysis of the Impact of Subprime Mortgage Crisis on China's Stock Market-Based on the Linkage Analysis of Sino-US Stock Market. Business Review, 21, 21-32.

[8] Fang, Y. and Gui, P. (2010) The Linkage of Stock Markets in Asia-Pacific Region-Based on the Study of Subprime Lending Impact. Word Economy Studies, No. 8, 22-32.

[9] Zhang, B., Fan, Z.Z. and Li, X.D. (2010) The Linkage Research of Chinese and American Stock Markets. Economic Research Journal, No. 11, 141-151.

[10] Podobnik, B. and Stanley, H.E. (2008) Detrended Cross-Correlation Analysis: A New Method for Analyzing Two Nonstationary Time Series. Physical Review Letters, 100, 084102. https://doi.org/10.1103/PhysRevLett.100.084102

[11] Zhou, W.X. (2008) Multifractal Detrended Cross-Correlation Analysis for Two Non-Stationary Signals. Physical Review E, 77, 066211. https://doi.org/10.1103/PhysRevE.77.066211

[12] Ma, F., Wei, Y. and Huang, D. (2013) Multifractal Detrended Cross-Correlation Analysis between the Chinese Stock Market and Surrounding Stock Markets. Physica $A$, 392, 1659-1670. https://doi.org/10.1016/j.physa.2012.12.010

[13] Wang, Y.D., Wei, Y. and Wu, C.F. (2010) Cross-Correlations between Chinese A-Share and B-Share Markets. Physica A, 389, 5468-5478. https://doi.org/10.1016/j.physa.2010.08.029

[14] Peters, E.E. (1994) Fractal Market Analysis: Applying chaos theory to Investment and Economics. John Wiley \& Son Inc., New York.

[15] Podobnik, B., Crosse, I., Horvati, D., et al. (2009) Quantifying Cross-Correlations Using Local and Global Detrending Approaches. The European Physical Journal B, 71, 243-250. https://doi.org/10.1140/epjb/e2009-00310-5 
[16] Zebende, G. (2011) DCCA Cross-Correlation Coefficient: Quantifying Level of Cross-Correlation. Physica A, 390, 614-618. https://doi.org/10.1016/j.physa.2010.10.022

[17] Yuan, Y. and Zhuang, X. (2009) Measuring Multifractality of Stock Price Fluctuation Using Multifractal Detrended Fluctuation Analysis. Physica A, 338, 2189-2197. https://doi.org/10.1016/j.physa.2009.02.026

[18] Shadkhoo, S. and Jafari, G.R. (2009) Multifractal Detrended Cross-Correlation Analysis of Temporal and Spatial Seismic Data. The European Physical Journal B, 72, 679-683. https://doi.org/10.1140/epjb/e2009-00402-2 